\title{
Relations Between Coefficients of Fractional Parentage
}

\author{
L. Zamick ${ }^{1}$ \\ ${ }^{1}$ Department of Physics and Astronomy, \\ Rutgers University, Piscataway, New Jersey 08854, USA
}

(Dated: July 15, 2018)

\begin{abstract}
For each of the $(9 / 2),(11 / 2)$ and $(13 / 2)$ single $\mathrm{j}$ shells we have only one state with $\mathrm{J}=\mathrm{j} v=3$ for a five particle system. For four identical particles there can be more than one state of seniority four. We note some "ratio" relations for the coefficients of fractional parentage for the four and five identical particle systems, which are found in the works of de Shalit and Talmi $[1,2]$ to be useful for explaining the vanishing of a five particle cfp. These relations are used to show that there is a special $\left(\mathrm{g}_{9 / 2}\right)^{4} \mathrm{I}=4 v=4$ wavefunction that cannot be admixed with an $\mathrm{I}=4 v=2$ wavefunction, even with seniority violating interactions.
\end{abstract}

PACS numbers: 


\section{INTRODUCTION}

In examining the Bayman-Lande tables [3] of coefficients of fractional parentage (cfp) we note some interesting relationships between (cfp)'s involving five nucleons with seniority three and four nucleons with seniority four. These results are of particular interest for the $g_{9 / 2}, h_{9 / 2}$, and $i_{13 / 2}$. For each of these shells there is only one state with total angular momentum $\mathrm{J}=\mathrm{j}$ and seniority $v=3$. For the four particle system for certain angular momenta I there are in some cases more than one state of seniority four. For example for the $\left(g_{9 / 2}^{4}\right)$

configuration there are two $(v=4 \mathrm{I}=4)$ states and two $(v=4 \mathrm{I}=6)$ states. For $\left(i_{13 / 2}\right)^{4}$ there are three $(v=4 \mathrm{I}=7)$ states, etc.

Let us designate the different $v=4$ states by $v_{i}$. We define

$$
C\left(v_{i}\right)=\left[j^{3}\left(J_{o}=j\right) j \mid j^{4} I v_{i}\right]
$$

and

$$
D\left(v_{i}\right)=\left[j^{4} I v_{i} j \mid j^{5}(J=j) v=3\right]
$$

where we use the standard notation for coefficients of fractional parentage.

The following relationships should be noted.

$$
\frac{C\left(v_{i}\right)}{C\left(v_{j}\right)}=\frac{D\left(v_{i}\right)}{D\left(v_{j}\right)}
$$

These relations have been explained in the books of deShalit and Talmi [1] and Talmi [2]. See especially Eq. (19.31) of ref. [2].

$$
\begin{aligned}
& {\left.\left[j^{v+1}\left(v+1, \alpha_{1} J_{1}\right) j J \mid\right\} j^{v+2} v \alpha J\right] } \\
= & (-1)^{J+j-J_{1}} \sqrt{\frac{2\left(2 J_{1}+1\right)(v+1)}{(2 J+1)(v+2)(2 j+1-2 v)}} \\
\times & {\left.\left[j^{v}(v \alpha J) j J_{1} \mid\right\} j^{v+1}\left(v+1, \alpha_{1}\right) J_{1}\right] }
\end{aligned}
$$

\section{Example}

We here list $g_{9 / 2}$ cfp table from Bayman and Lande [3] for the case $\mathrm{n}=4, \mathrm{I}=4$ and $v=4$. 


\begin{tabular}{|c|c|c|c|c|}
\hline \multicolumn{5}{|c|}{ Table $1\left(9 / 2^{4}\right)$} \\
\hline$v_{3}$ & $\mathrm{~J} 1$ & $v_{1}=4$ & $v_{2}=4$ & $v_{a}=4$ \\
\hline \hline 3 & $3 / 2$ & -0.080040 & 0.482164 & -0.12219 \\
\hline \hline 3 & $5 / 2$ & 0.020346 & -0.391860 & -0.05477 \\
\hline \hline 3 & $7 / 2$ & -0.620338 & -0.010083 & -0.61704 \\
\hline \hline 1 & $9 / 2$ & 0. & 0. & 0. \\
\hline \hline 3 & $9 / 2$ & 0.030755 & 0.347913 & 0. \\
\hline \hline 3 & $11 / 2$ & 0.431655 & 0.291285 & 0.40432 \\
\hline \hline 3 & $13 / 2$ & 0.594545 & -0.256411 & 0.61481 \\
\hline \hline 3 & $15 / 2$ & 0.205016 & 0.505621 & 0.15975 \\
\hline \hline 3 & $17 / 2$ & 0.159916 & -0.295249 & 0.18529 \\
\hline \hline \multicolumn{5}{|c}{}
\end{tabular}

$\mathrm{I}=4 \quad \mathrm{j}=9 / 2$

From Table 1 for 4 particles and for the 5 particle listing in Bayman-Lande [3] for a $\mathrm{J}=\mathrm{j}$, $v=3$ state.

$\mathrm{I}=4(9 / 2)^{4} \quad \mathrm{~J}=\mathrm{j}(9 / 2)^{5}$

$\mathrm{C}\left(v_{1}\right)=0.030755 \quad \mathrm{D}\left(v_{1}\right)=-0.018452$

$\mathrm{C}\left(v_{2}\right)=0.347913 \quad \mathrm{D}\left(v_{2}\right)=-0.208747$

Ratio $11.312 \quad$ Ratio 11.312

It was noted in ref. [4] that in the techniques used to calculate cfp's in ref. [3] the two $\mathrm{t}=4 v=4$ states $\left(v_{1}\right.$ and $\left.v_{2}\right)$ were degenerate. Hence the emerging $v_{1}$ and $v_{2}$ were somewhat arbitrary. Any linear combination of the two would be equally valid.

$$
\begin{aligned}
& v_{a}=\alpha v_{1}+\beta v_{2} \\
& v_{b}=-\beta v_{1}+\alpha v_{2}
\end{aligned}
$$

If instead of a pairing interaction, one uses a seniority conserving interaction with nondegenerate levels e.g. the delta interaction, then one of the linear combinations, say $v_{a}$ has 
the interesting property that it cannot mix with a $v=2$ state via a seniority non-conserving interaction

$$
M=<I=4 v=2|V| I=4 v_{a}>=0
$$

The cfp's for the state $\mathrm{I}=4 v_{a}$ are listed in the last column of Table 1.

Note that both the cfp's for $v_{o}=1$ and $v_{o}=3 \mathrm{~J}=\mathrm{j}=9 / 2$ vanish.

Because of the proportionality relationship between 4 particle cfp's and 5 particle cfp's (2) we have the result

$$
\left.\left[j^{4} I=4 v_{a} \mid\right\} j^{5} J=j v=3\right]=0
$$

At mid-shell the $v=3$ state does not mix with $v=1$ or $v=5$. This point is crucial for the next section.

\section{FURTHER RELATIONS}

We can write

$$
M=\sum_{J_{A}} M\left(J_{A}\right) E\left(J_{A}\right)
$$

where $\mathrm{E}\left(\mathrm{J}_{A}\right)$ is the two body matrix element

$$
E\left(J_{A}\right)=<\left(j^{2}\right) J_{A} V\left(j^{2}\right) J_{A}>
$$

In order to prove that $\mathrm{M}$ vanishes for any interaction we must show that

$$
M\left(J_{A}\right)=0 \quad J_{A}=0,2,4,6,8
$$

We can get an expression for $\mathrm{M}$ in two steps. First we eliminate one of the four particles and get the expression in terms of a three particle interaction. This involves a sum over a three particle angular momentum $\mathrm{J}_{3}$ and seniority $v_{3}$. However, only terms with $J_{3}=j v_{3}$ $=3$ will contribute so we supress this index. 
We obtain

$$
\begin{aligned}
M= & \left.(4 \times 3 / 2) \sum_{J_{3}}\left[j^{3} J_{3} j \mid\right\} j^{4} I v=2\right] \\
& {\left.\left[j^{3} J_{3} j \mid\right\} j^{4} I v_{a}=4\right]<\left(j^{3}\right)^{J_{3}} V\left(j^{3}\right)^{J_{3}}>}
\end{aligned}
$$

The three body matrix element can now be expressed in terms of two body matrix elements

$$
<\left(j^{3}\right) J_{3} V\left(j^{3}\right) J_{3}>=\sum_{J_{A}}\left[\left(j^{2}\right) J_{A} j \mid j^{3} J_{3}\right]^{2} E\left(J_{A}\right)
$$

We now note the following $\mathrm{n}=2$ Redmond relation [5] as modified by Zamick and Escuderos $[6]$.

$$
\sum_{v_{3}}\left|\left(j^{2} J_{A} j \mid\right\} j^{3} J_{3} v_{3}\right|^{2}=1 / 3+2 / 3\left\{\begin{array}{ccc}
j & j & J_{A} \\
J_{3} & j & J_{A}
\end{array}\right\}\left(2 J_{A}+1\right)
$$

We finally get

$$
\begin{aligned}
M\left(J_{A}\right)= & \left.6 \sum_{J_{3}}\left[j^{3} J_{3} j \mid\right\} j^{4} I=4 v=2\right] \\
& {\left.\left[j^{3} J_{3} j \mid\right\} j^{4} I=4 v_{a}=4\right] } \\
& {\left[1 / 3+2 / 3\left\{\begin{array}{lll}
j & j & J_{A} \\
J_{3} & j & J_{A}
\end{array}\right\}\left(2 J_{A}+1\right)\right] }
\end{aligned}
$$

for $J_{A}=0,2,4,6$ and 8 .

The " $1 / 3$ " term above will vanish only if the sum $J_{3}$ can be taken over all possible values from $3 / 2$ to $17 / 2$. This is only true for $J_{A}=4$ and $J_{A}=6$.

For $J_{A}=4$ we can use a "4-5" Redmond relation [5] (also eq. 7 and 8 of ref. [6]) to obtain

$$
\begin{array}{r}
\left.M\left(J_{A}=4\right)=5\left[j^{4}(I=4 v=2), j \mid\right\} j^{5} J=j v=3\right] \\
\left.\left[j^{4}\left(I=4 v_{a}=4\right), j \mid\right\} j^{5} J=j v=3\right]
\end{array}
$$

However we have shown in Eq. 5 the following

$$
\left.\left[j^{4}\left(I=4 v_{a}=4\right), j \mid\right\} j^{5} J=j v=3\right]=0
$$


Hence we have

$$
M\left(J_{A}=4\right)=0
$$

We can use this fact to prove the result of Eq. (4) i.e. that $M=0$. Recall that in the $f_{7 / 2}$ shell there is no seniority mixing for any interaction. One way of proving this is to note that one can find three independent seniority conserving interactions. In the $\mathrm{f}_{7 / 2}$ shell there are four identical particles there are four two body matrix elements $\mathrm{J}_{A}=0,2,4$ and 6 . However, we can always add an arbitrary constant to get $\mathrm{E}\left(\mathrm{J}_{A}=0\right)=0$, so that there are only three. In the $\mathrm{g}_{9 / 2}$ shell there are four matrix elements, $\mathrm{J}_{A}=2,4,6$ and 8 .

The three previously mentioned seniority conserving interactions (pairing, delta and $\mathrm{j}(1)$ - $\mathrm{j}(2))$ will not admix seniority $v=2$ and $v=4$ states. But we have just found a seniority violating interaction which will not admix $v=2$ and the $v_{a}=4$ state. This latter interaction is

$$
<\left(j^{2}\right) J_{A} V\left(j^{2}\right) J_{A}>=V_{o} \delta_{J_{A}, 4}
$$

Hence, we can express the four matrix elements in terms of these four interactions which will not admix the $v_{a}=4$ state with the $v=2$ state. Indeed all $\mathrm{M}\left(J_{A}\right)$ vanish.

Using the same arguments that were given in Eq. (13), (14) and (15) we can show that there is no coupling via the interaction (16) $\left(\mathrm{J}_{A}=4\right.$ pairing) between the state. $\mathrm{I}=4 \mathrm{~V}_{a}=4$ and the $\mathrm{I}=4 \mathrm{v}=4$ state that is orthogonal to it. This has yet to be shown for a general interaction.

We next consider the energy of the $\mathrm{v}_{a}=4$ state. Using mathematica, an expression for the energy was obtained by P. Van Isacker and S. Heinz [7].

$$
E\left((9 / 2)^{4}, I=4 v_{a}=4\right)=\frac{68}{33} E(2)+E(4)+\frac{13}{15} E(6)+\frac{114}{55} E(8)
$$

with minor modifications of what has been done up to now, we can explain why the coefficient of $\mathrm{E}(4)$ is one. We can write

$$
E\left(j^{4}, I=4 v_{a}=4\right)=\sum_{J_{a}} G\left(J_{A}\right) E\left(J_{A}\right)
$$

We can get $\mathrm{G}\left(\mathrm{J}_{A}\right)$ from Eq. (12) by replacing the $\mathrm{v}=2$ cfp by the $\mathrm{v}_{a}=4$ cfp. Hence we obtain 


$$
\left.G\left(J_{A}\right)=6 \sum_{J_{A}}\left[j^{3} J_{3} j \mid\right\} j^{4} I=4 v_{a}=4\right]^{2}\left[\frac{1}{3}+\frac{2}{3}\left\{\begin{array}{ccc}
j & j & J_{A} \\
j_{3} & j & J_{A}
\end{array}\right\}\left(2 J_{A}+1\right)\right]
$$

For $\mathrm{J}_{A}=4$ we can again use the Redmond relation [5] but care must be taken because we now have a diagonal term. Also the " $1 / 3$ " term now gives a contribution. We obtain

$$
G(4)=6[1 / 3+2 / 3(-1 / 4)]+\text { vanishing } \text { cfps }=1
$$

\section{APPENDIX: THE REDMOND RECURSION RELATION IN THE SENIOR- ITY SCHEME}

We here present the equivalent of the Redmond resursion relation, but for cfp's classified by the seniority quantum number $v$ and for which there are no redundacies [6]. Here is our formula:

$$
\begin{aligned}
& \left.(n+1) \sum_{v_{s}}\left[j^{n}\left(v_{0} J_{0}\right) j I_{s}\right]\left[j^{n}\left(v_{1} J_{1}\right) j I_{s} \mid\right\} j^{n+1} v_{s} I_{s}\right]= \\
= & \delta_{J_{0} J_{1}} \delta_{v_{0} v_{1}}+n(-1)^{J_{0}+J_{1}} \sqrt{\left(2 J_{0}+1\right)\left(2 J_{1}+1\right)} \sum_{v_{2} J_{2}}\left\{\begin{array}{lll}
J_{2} & j & J_{1} \\
I_{s} & j & J_{0}
\end{array}\right\} \\
\times & {\left.\left.\left[j^{n-1}\left(v_{2} J_{2}\right) j J_{0} \mid\right\} j^{n} v_{0} J_{0}\right]\left[j^{n-1}\left(v_{2} J_{2}\right) j J_{1} \mid\right\} j^{n} v_{1} J_{1}\right] . }
\end{aligned}
$$

Note that we sum over all final seniorities but the final total angular momentum is fixed.

I thank Igal Talmi and Piet Van Isacker for helpful discussions. I thank the INT-Seattle where some of the added work was done.

[1] A. deShalit and I. Talmi, Nuclear Shell Theory, (Academic Press, New York, 1963).

[2] I. Talmi, Simple Models of Complex Nuclei (Harwood Academic, Reading U.K., 1993).

[3] B. F. Bayman and A. Lande, Nucl. Phys. $\underline{77}, 1$ (1966).

[4] A. Escuderos and L. Zamick, Phys. Rev. $\underline{\text { C73 }}, 044302$ (2006).

[5] P. Redmond, Proc. R. Soc. London $\underline{\mathrm{A} 222}, 84$ (1954).

[6] L. Zamick and A. Escuderos, Annals of Physics, $\underline{321}, 987$ (2006).

[7] P. Van Isacker and S. Heinz, private communication, Yale 2007 Nuclear Physics Workshoppower point presentation online. 